\title{
Antagonist design through forced electrostatic mismatch
}

The structure of an Interleukin-4 mutant shows that an uncompensated charge disables the interaction between cytokine and low-affinity receptor.

Sir - The binding of cytokines to their receptors is governed by typical protein-protein interactions, which determine the specificity and kinetics of the recognition process'. Antagonists of cytokines are variants
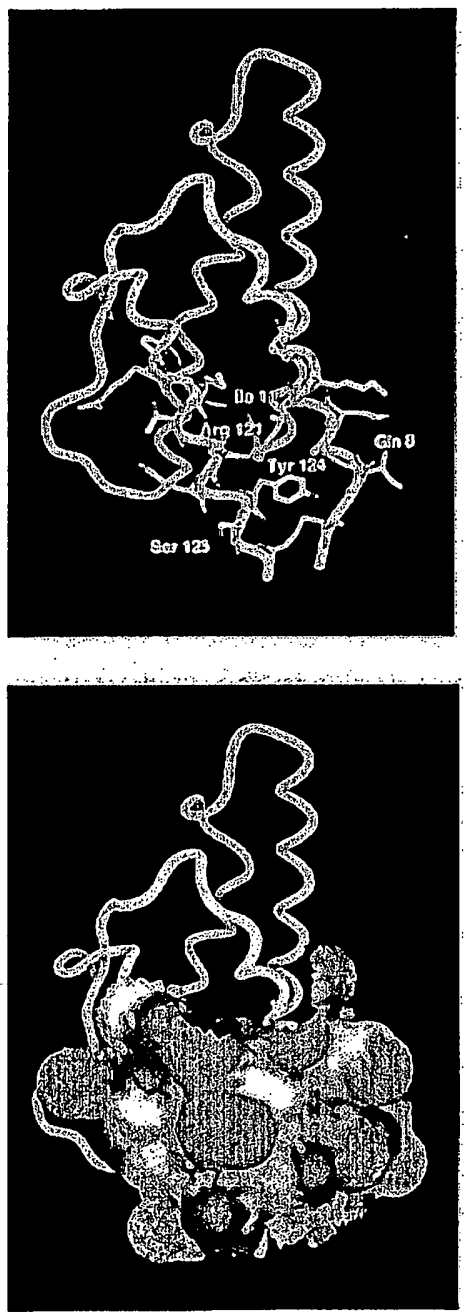

which bind tightly to one receptor subunit without causing receptor oligomerization. Design of cytokine antagonists may be the most rational way to suppress specific signalling pathways.
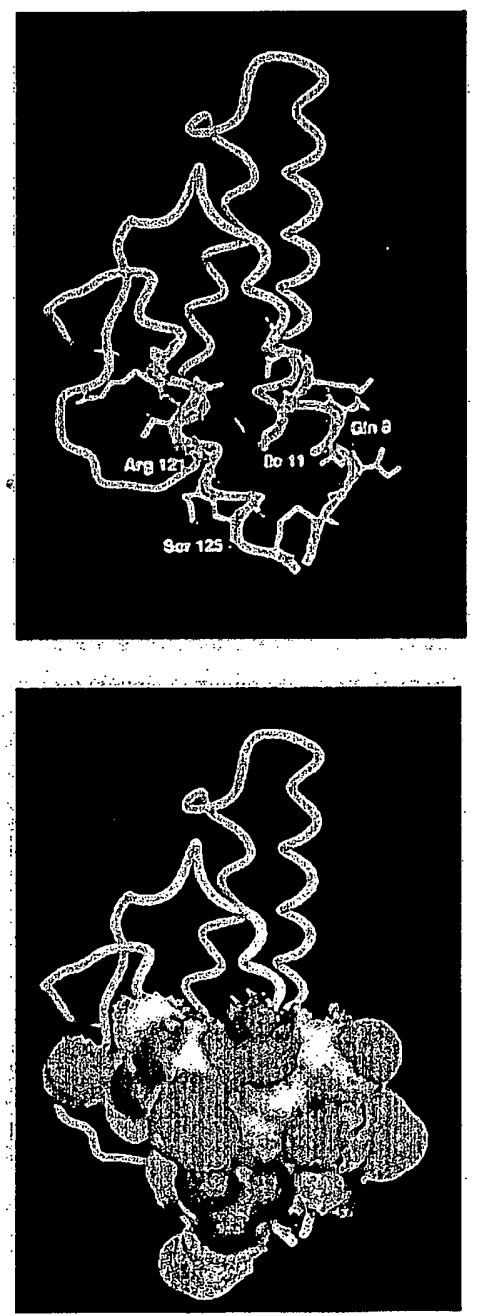

Interleukin 4 (IL-4) is a typical cytokine with a broad range of biological activities on various cell types of the lymphoid system (for recent reviews see refs 2,3 ). This cytokine is well characterised structurally ${ }^{+-9}$

Fig. 1 Backbone model (top) and solvent accessible surfaces of the binding epitope (bottom) of the averaged minimized structures of $a$, wild-type IL-4, $b, Y 124 G$ and $c, Y 124 D$. In the backbone model, the sidechains of C3, 15, Q8, G9, $111, \mathrm{~K} 12, \mathrm{~N} 15, \mathrm{Q} 114$, $R 115, K 117, T 118, R 121, Q 122, X 124, S 125$, and C127 are included. The solvent accessiblity was calculated using a $1.4 A$ radius rolling probe in the program GRASP. The epitope which is majorly responsible for the binding of the second receptor subunit is indicated by a circle. Nitrogen atoms are drawn in blue, oxygen atoms in red. 


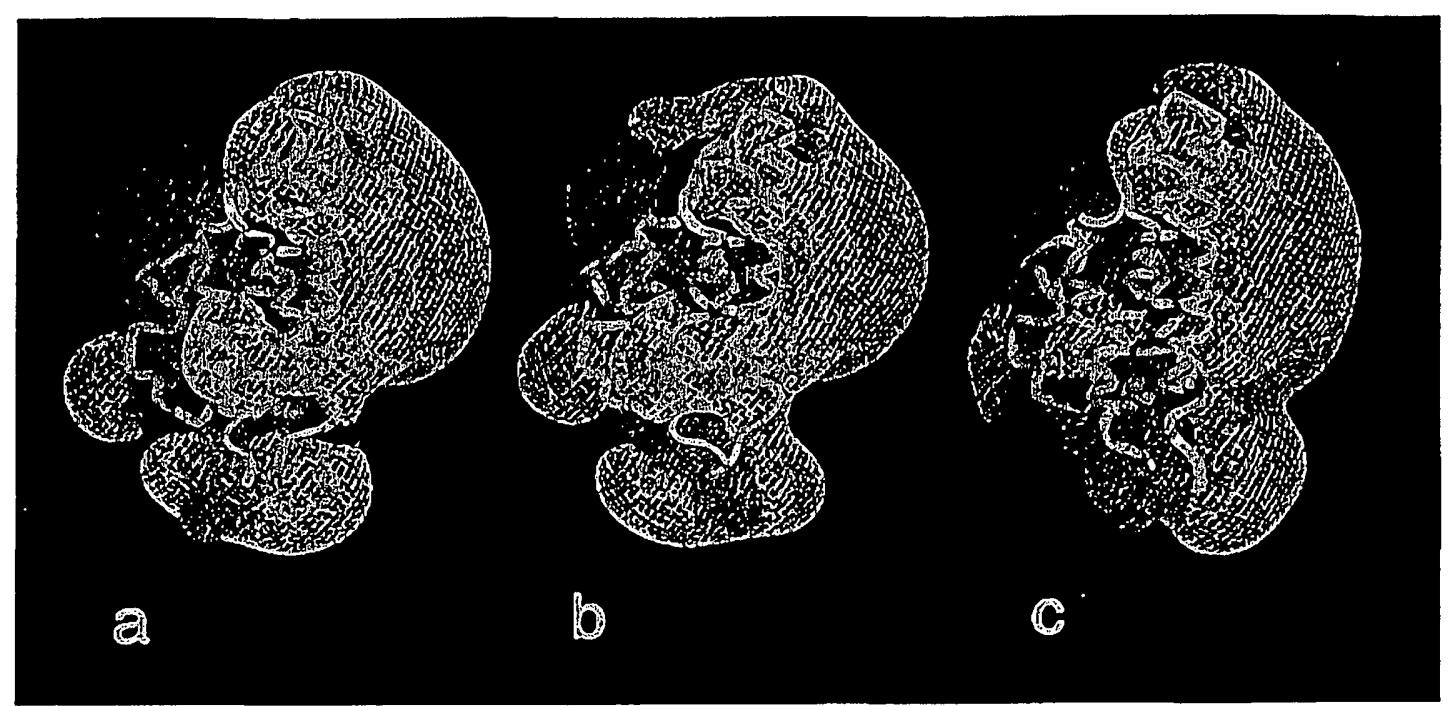

Fig. 2 Electrostatic potentials of the a, IL-4 wild-type, b, Y124G and $c, Y 1240$ as calculated by numerically solving the finite difference linearized Poisson-Boltzmann equation using the program UHBD, version 4.0 (ref. 21). The OPLS parameter set (ref. 22), with the radii of hydrogen atoms set to $1.2 \AA$, was used to assign atomic radii and partial charges. Dielectric constants of 78 and 2 were assigned to the solvent and the solute respectively. The solvent-solute dielectric boundary smoothing was implemented ${ }^{23}$. the ionic strength of the solvent was set to $145 \mathrm{mM}$ and to follow a Boltzmann distribution at $300 \mathrm{~K}$. A 2 A exclusion layer was used. A grid with a $1 \AA$ spacing was taken for the calculations. The grids were contoured at $+1-0.3$

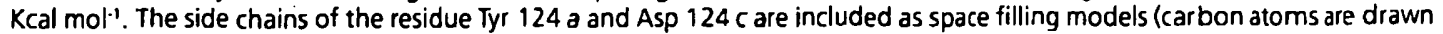
in green, oxygen atoms in red).

and appears to be involved in the specific expression of IgE low-affinity receptor (FcERII, CD23). It also acts as an isotype switching factor mediating the switch from IgM to IgG1 and IgE expression. Its involvement in allergic diseases makes IL-4 an important target for drug design. Although a homodimeric IL-4/IL-4R complex has been modelled by several groups ${ }^{10.11}$, it has recently been proposed that the $\gamma$-chain of the IL2 receptor is involved in the IL- 4 system ${ }^{12-13}$. IL-4, IL-2 and human growth hormone (hGH) were the first cytokines for which point mutants with antagonistic profiles were reported ${ }^{14-16}$. These antagonists function differently. In the case of $\mathrm{hGH}$, a steric block to the binding of the second receptor unit was introduced by the substitution of glycine to a tryptophan residue ${ }^{16}$. In contrast, the IL-4 variant Y124D is an efficient antagonist although the side chain of tyrosine, is replaced by a sterically less demanding one, aspartic acid. The variant Y124G shows a strongly reduced agonism; only $20 \%$ of the activity remains. Similar observations have been made for IL2 (ref. 15). Such a large activity change due to the substitution of one residue seems paradoxical in view of the large binding area between the hormone and the receptor subunit ${ }^{17}$. Other studies have suggested that the activity changes are due to large structural changes in the mutant protein ${ }^{6,10.11}$

To address this question, we have derived the structures of the mutants Y124G and Y124D in solution by nuclear magnetic resonance spectroscopy. The structures were calculated using a hybrid distance geom. etry-simulated annealing protocol ${ }^{18}$ and the program Xplor ${ }^{19}$. The mutant structures were calculated on the basis of 998 NOEs (Y124D) and 973 NOEs (Y124G) (see Table la); the data were taken from NOESY spectra with mixing times of 20,40 and $80 \mathrm{~ms}$ (ref. 9).

The structures of the mutants Y124G and Y124D are very similar to those of the wild-type protein. The root-mean-square deviation between the $\mathrm{X}$-ray structure, $1 \mathrm{rcb}$, and our mutant structures is $1.36 \AA$ ( $Y 124 G)$ and $1.53 \AA$ (Y)24D) $(C \alpha$, $\mathrm{N}, \mathrm{C}^{\prime}$ of residues 5-125). Very similar values are observed for the other NMR structures $^{9}$ (see Table I $b$ ).

The r.m.s.d. for residues 5-19 and
109-125 (helix $A$ and $D$ ) between $1 \mathrm{rcb}$ and $Y 124 \mathrm{G}$ is $0.88 \AA$ and for $Y 124 D$ is $0.96 \AA$. This shows that the effect of the mutations and hence the reason for the antagonistic activity is not in the unfolding of helices $D$ and $A$ (refs $6,10,11$ ). The decreasing agonism must therefore be attributed to changes in the electrostatic potential or to changes in the hydrophobicity of the surface. A detailed inspection of the mutation site in the wild type and the variants shows that the epitope's structure remains intact. The $\delta$-carbon of the aspartic acid residue in the mutant Y124D superimposes with the $\gamma$-carbon of the tyrosine ring in the wild-type protein. The side chain of Arg 121 is ordered up to the $\delta$-carbon in all three structures, although the position of the guanidinium group cannot be determined. The side chain of Ser 125 is in a very similar position in all three structures. The position of the a mide group of the side chain of Glu 8 varies, its whole side chain is protruding straight into solution.

A detailed comparison of the binding epitope surface between the IL-4 wild-type and the Y124G and Y124D variants is shown in Fig. 1. 
The importance of the hydrophobic area made up by the aromatic ring of Tyr 124 is supported by the finding that a phenylalanine or a histidine in place of the tyrosine shows the same activity as the wild-type ${ }^{14}$, but the Gly mutant (and Y124N or Y124K) shows an activity of only $20 \%$ in the T-cell proliferation test. This indicates that this loss of activity can be directly attributed to the loss of approximately $60 \AA^{2}$ of hydrophobic surface (Fig. 1b). Estimating a binding energy of $20 \mathrm{cal} \AA^{-2}$ for hydrophobic interactions $s^{20}$, the deletion' of a hydrophobic surface of this size should result in a reduction of binding energy of about $1 \mathrm{kcal}$ mol $^{-1}$, in agreement with results obtained by Cunningham and Wells ${ }^{1}$. The activity decreases further with the introduction of a negatively charged side chain in the antagonist Y124D (Fig. 1c). In this case, the binding surface is not dramatically reduced, but now a strongly negatively charged group appears in place of the tyrosine ring.

Calculations of the electrostatic properties at the mutation site show that the wild-type protein exposes a slightly positive potential in this area, which is changed to a negative charge through the carboxyl group of the aspartate side chain (Fig. $2 a-c$ ). Although the electrostatic interactions may contribute less to the binding strength than hydrophobic interactions ${ }^{1}$, the repulsive forces introduced by a mismatch of the electrostatic potentials must be large and cannot be compensated for by other interactions involved in the binding of the second receptor subunit, and hence reduce the activity further ${ }^{24}$.

\begin{tabular}{lccc}
\hline & \multicolumn{3}{c}{ Table 1a } \\
\hline Type of NOE & wild-type IL-4 & Y124G & Y124D \\
intra-residue & 259 & 273 & 272 \\
sequential & 391 & 347 & 363 \\
medium-range & 172 & 156 & 158 \\
long-range & 224 & 197 & 205 \\
total & 1046 & 973 & 998
\end{tabular}

medium-range NOE: $(i, j), i+2 \leq j \leq i+4$

long-range NOE: $\quad(i, j), j \geq i+5$

\begin{tabular}{lccc}
\hline \multicolumn{4}{c}{ Table $\boldsymbol{b} \boldsymbol{b}$} \\
\hline atoms used in alignment & Ircb - IL-4 & Ircb - Y124G & Ircb - Y124D \\
5-125 backbone & 1.58 & 1.36 & 1.53 \\
heavy atoms & 2.46 & 2.32 & 2.37 \\
& & 0.88 & 0.96 \\
5-19 and 109-125, backbone & 0.74 & 1.93 & 1.91 \\
heavy atoms & 1.80 & 0.73 & 0.53 \\
& 0.50 & 1.97 & 1.78 \\
109-125 backbone & 1.78 & & \\
heavy atoms & & 1.21 & 1.29 \\
5-19 and 109-125, bb plus & 1.21 & & \\
heavy atoms of core- & & &
\end{tabular}

backbone atoms $=C \alpha, N$ and $C^{\prime}$

* Heavy atoms of residue types isoleucine, leucine, phenylalanine, tyrosine and methionine were used.

In summary, this work shows that an effective four helix bundle cytokine antagonist can be designed by the removal of a larger hydrophobic area and the introduction of an electrostatic mismatch with the receptor.

Received 9 June; accepted 26 August, 1994.

Thomas Müller', Walter Sebald ${ }^{1}$ and Hartmut Oschkinat
European Molecular Biology Laboratory

Meyerhofstr. 1, D-69012 Heidelberg, Germany

'Theodor-Boveri-Institut für Biowissenschaften (Biozentrum) der Univers̆t Würzburg, Physiologische Chemie II, Am Hubland, D-97074 Würzburg, Germany

Correspondence should be addressed to W.S. or H.O.
1. Cunningham, B.C. \& Wells, J.A. J molec Biol. 234, 554-563 (1993).

2. Boulay, J.A. \& Paul, W.E. Curr. Opin immun. 4, 294-298, (1992)

3. Paul, W.E. \& Seder, R.A. Cell 76, 241-251 (1994)

4. Redfield, C., Boyd, J. , Smith, L.J., Smith, R.A.G. \& Dobson, C.M. Biochemistry 31 , 10431-10437 (1991).

5. Smith, I.J. et al. J. molec. Biol. 224, 899 904 (1992).

6. Powers, R. et al., Biochemistry 32, 6744 6762 (1993).

7. Walter, M.R. et al., J. biol. Chem. 267. 20371-20376 (1992)

8. Wlodawer. A., Pavlovsky. A. \& Gustchina,

A. FEBS Lett. 309, 59-64 (1992).

9. Muller, T., Dieckmann, T., Sebald, W. \&
Oschkinat, H. J. molec. Biol. 237, 423-436 (1994).

10. Bamborough, P., Grant, G.H., Hedgecock C.J.R., West, S.P. \& Richards, W.G. Proteins 17, 11-19 (1993)

11. Wlodawer, A., Pavlovsky, A. \& Gustchina, A. Prot. Sci. 2, 1373-1382 (1993).

12. M. Kondo et al. Science 262, 1874-1877 (1993).

13.S.M. Russell et al. Science 262, 1818 (1993).

14. Kruse, N., Tony, H.-P. \& Sebald, W. EMBO J. 11, 3237-3244 (1992)

15. Zurawski, S.M. \& Zurawski, G. EMBO I. 11. 3905-3910 (1992)

16. Fuh, G. et al. Science 256, 1677-1680 (1992).
17. de Vos, A.M. Ulisch, M. \& Kossiakoff A.A. Science 255, 306-312 (1992).

18. Nilges, M., Clore, G.M. \& Gronenborn, A FEBS Letters 229, 317-324 (1988).

19. Brünger, A.T. Xplor Version 3.0 Manual (1992), Yale University, New Haven,

20. Richards, F.M. Annu. Rev. biophys. Bioeng 6. 151-176 (1977)

21. Davis, M.E., Madura, J.D., Luty, B.A. \& MCCammon, J.A. J. comp. Phys. Commun. 62. 187-197 (1990).

22. Jorgensen, W.L. \& Tirado-Rives, J. J. Am. chem. Soc. 110, 1657-1666 (1988)

23. Davis, M.E. \& MCCammon, J.A. J. comp. Chem. 7. vol. 12, 909-912 (1991).

24. Rogers, N.K. Prog. Biophys. molec Biol. 48, 37-66 (1986). 